\title{
Oligodendroglioma with Sarcomatous Transformation: Case Report and Literature Review
}

\section{Oligodendroglioma com transformação sarcomatosa: Caso clínico e revisão da literatura}

\author{
Gonçalo Cerdeira Figueiredo ${ }^{1}$ Célia Maria Pinheiro ${ }^{1}$ Alfredo Luís Calheiros ${ }^{1}$ \\ ${ }^{1}$ Department of Neurosurgery, Centro Hospitalar do Porto, Porto, \\ Address for correspondence Gonçalo Cerdeira Figueiredo, MD, \\ Portugal \\ Arq Bras Neurocir 2019;38:128-136. \\ Departmento de Neurocirurgia, Centro Hospitalar do Porto, Largo \\ Prof. Abel Salazar, 4099-001, Porto, Portugal \\ (e-mail: gfigueiredo03@gmail.com).
}

\begin{abstract}
Oligodendrogliomas are infiltrative tumors of the central nervous system considered to be morphologically stable and to offer a better prognosis. Here, we describe the case of a 36year-old man with an initial diagnosis of oligodendroglioma, World Health Organization

Keywords

- oligodendroglioma

- oligosarcoma

- co-deletion $1 \mathrm{p} / 19 \mathrm{q}$

- spinal metastasis (WHO) grade II, who presented transformation to a sarcomatous form, while maintaining the oligodendroglial component as well as the genetic characteristics of the initial tumor without having undergone any complementary treatments previously. Despite the favorable genetic characteristics, the tumor presented poor response to complementary treatments, and rapid progression, including spinal metastasis.

\section{Resumo}

Palavras-chave

- oligodendroglioma

- oligossarcoma

- co-deleção 1p/19q

- metástases raquidianas

Oligodendrogliomas são tumores infiltrativos do sistema nervoso central considerados morfologicamente estáveis e com melhor prognóstico. Aqui, descrevemos o caso de um homem de 36 anos com diagnóstico inicial de oligodendroglioma grau II OMS, que apresentou transformação para uma forma sarcomatosa, mantendo as características genéticas do tumor inicial, sem ter sido submetido a tratamento complementar. Apesar das características genéticas favoráveis, o tumor apresentou má resposta aos tratamentos e progressão rápida, incluindo com metastização raquidiana.
\end{abstract}

\section{Introduction}

Oligodendrogliomas are infiltrative tumors of the central nervous system (CNS). They constitute between 4 and $5 \%$ of the primary CNS tumors and $\sim 4$ to $15 \%$ of the glial tumors. ${ }^{1}$ Among the glial tumors, oligodendrogliomas have always been considered morphologically stable. ${ }^{2}$

Gliosarcomas are rare primary tumors of the CNS, histologically composed of glial and sarcomatous cells, with the glial component being, in most cases, astrocytic. ${ }^{3}$

(D) Gonçalo Cerdeira Figueiredo's ORCID is https://orcid.org/00000001-6623-6348.

received

October 23, 2018

accepted

February 19, 2019
DOI https://doi.org/

10.1055/s-0039-1685150. ISSN 0103-5355.
We describe here a case of an oligosarcoma (World Health Organization [WHO] grade $\mathrm{III}^{4}$ ), originating from an oligodendroglioma (WHO grade $\mathrm{II}^{4}$ ), in a patient not previously submitted to complementary treatments.

\section{Case Report}

A 36-year-old man presented with an onset of convulsive crisis in 2014. He underwent brain computed tomography (CT) that showed a left frontal intra-axial lesion with calcifications in the interior (-Fig. 1) and brain magnetic resonance imaging (MRI) that showed a lesion at the same location with hyperintensity T2 and without contrast

Copyright @ 2019 by Thieme Revinter

Publicações Ltda, Rio de Janeiro, Brazil

License terms

c) $(1) \$$ 


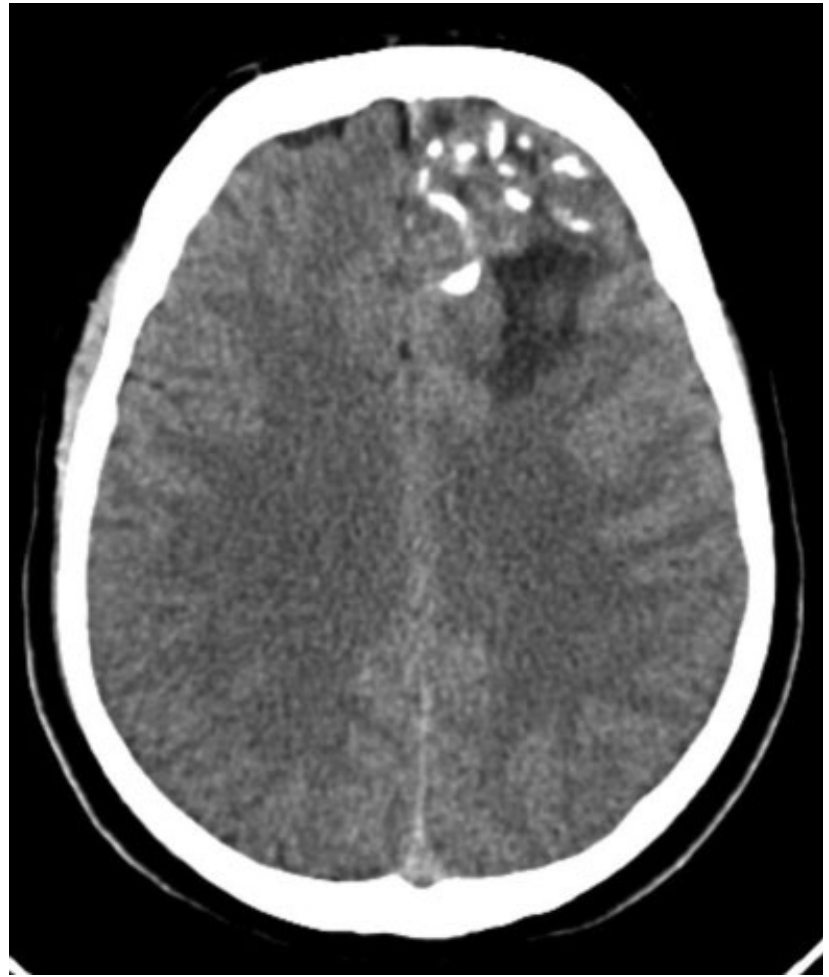

Fig. 1 Computed tomography scan showed a left frontal lesion with calcification.

enhancement, suggestive of low-grade glioma (-Fig. 2). The patient was submitted to craniotomy and gross total resection (GTR) of the lesion (-Fig. 3). Histological analysis showed a tumor with round nuclei cells and perinuclear

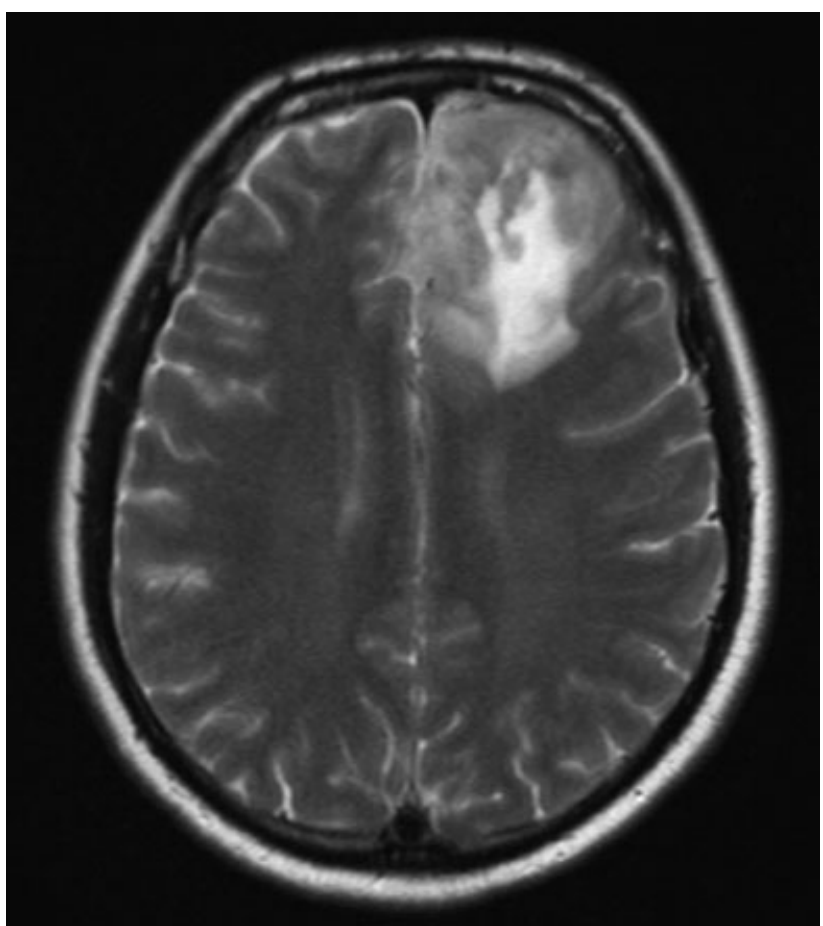

Fig. 2 T2-weighted magnetic resonance imaging showed a hyperintensity left frontal lesion.

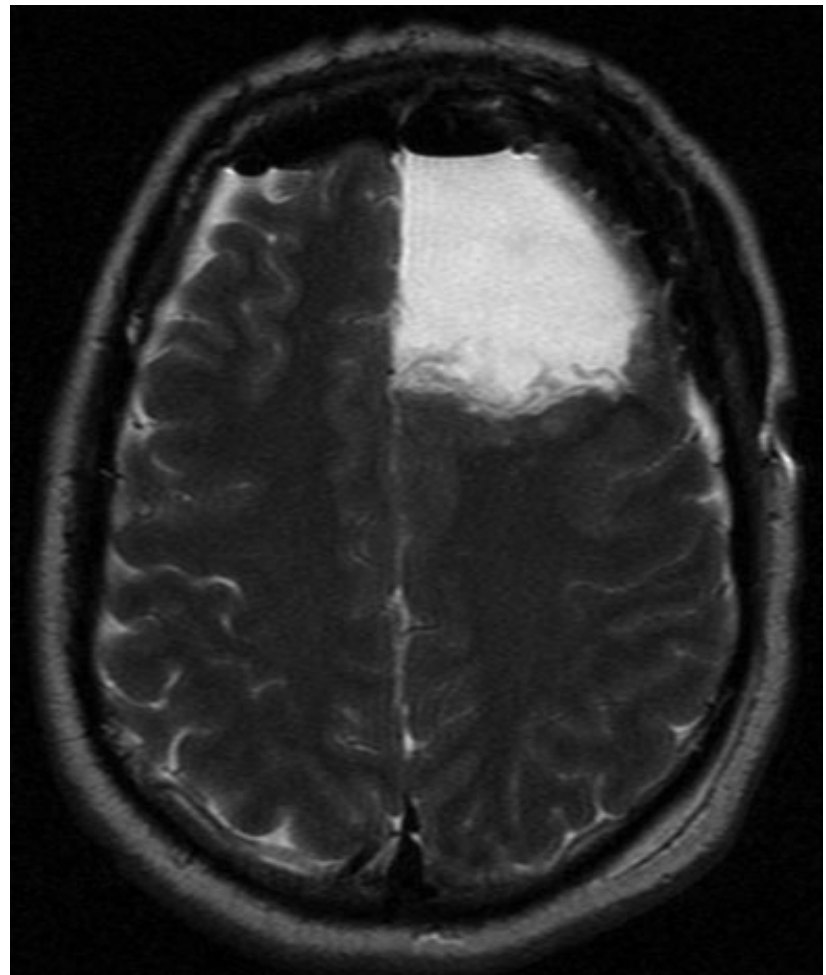

Fig. 3 First surgery postoperative magnetic resonance imaging showed gross total resection of the tumor.

halos, with extensive areas of calcification. It also showed absence of mitoses, necrosis and showed low nuclear proliferation index. The lesion was classified as oligoastrocytoma WHO grade II, according to the WHO tumor classification of $2007^{5}$ (-Figs. 4 and 5), and the tumor was positive for the presence of $1 \mathrm{p} / 19 \mathrm{q}$ co-deletion. The patient remained under clinical and imaging surveillance, without any complementary treatment.

Two years later, in imaging control, tumor growth was recorded in the posterior and medial portion of the surgical site. An MRI showed characteristics similar to the initial lesion, namely without evidence of contrast enhancement,

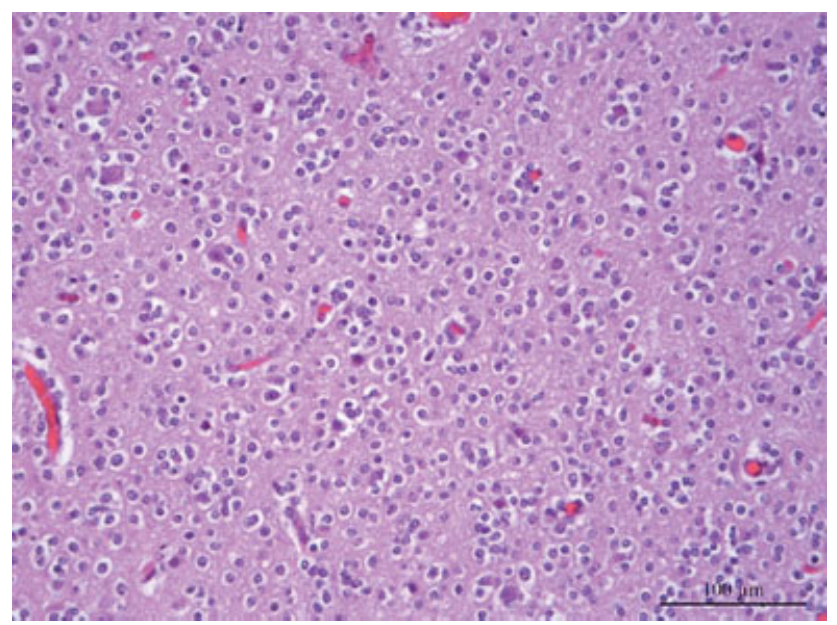

Fig. 4 HE staining showed round nuclei with a perinuclear halo, and a low proliferation index. 


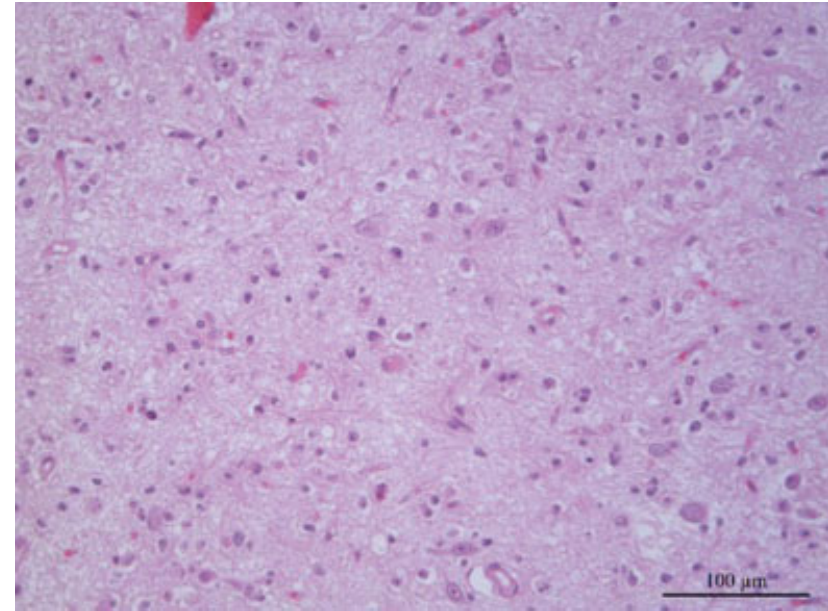

Fig. 5 HE staining showed round nuclei with a perinuclear halo, and a low proliferation index.

with spontaneous hyperintensity in T2-fluid-attenuated inversion recovery (FLAIR) weighted imaging (- Figs. 6 and 7). The patient underwent a new surgical procedure with GTR of the recurrent tumor ( - Fig. 8). The neuropathological characteristics confirmed that it was an oligodendroglioma WHO grade II, isocitrate dehydrogenase (IDH-1) positive (according to the WHO classification of 2016 CNS tumors ${ }^{4}$ ), maintaining the presence of $1 \mathrm{p} / 19 \mathrm{q}$ co-deletion and $\alpha$ thalassemia/mental retardation syndrome X-linked (ATRX) mutation positive (-Figs. 9 and $\mathbf{1 0}$ ), now with a moderate nuclear proliferation index. The patient was once again referred to clinical and imaging surveillance, not considering

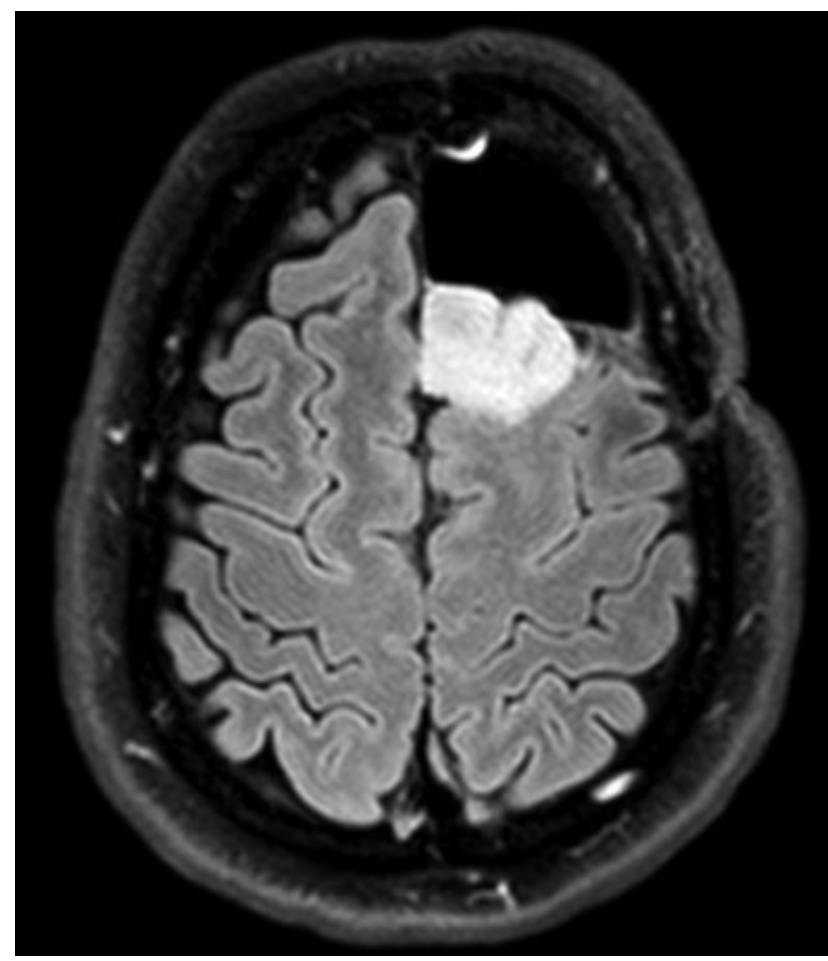

Fig. 6 T2-fluid-attenuated inversion recovery (FLAIR) magnetic resonance imaging showed a tumor recurrence in the posterior and medial walls of the surgical site.

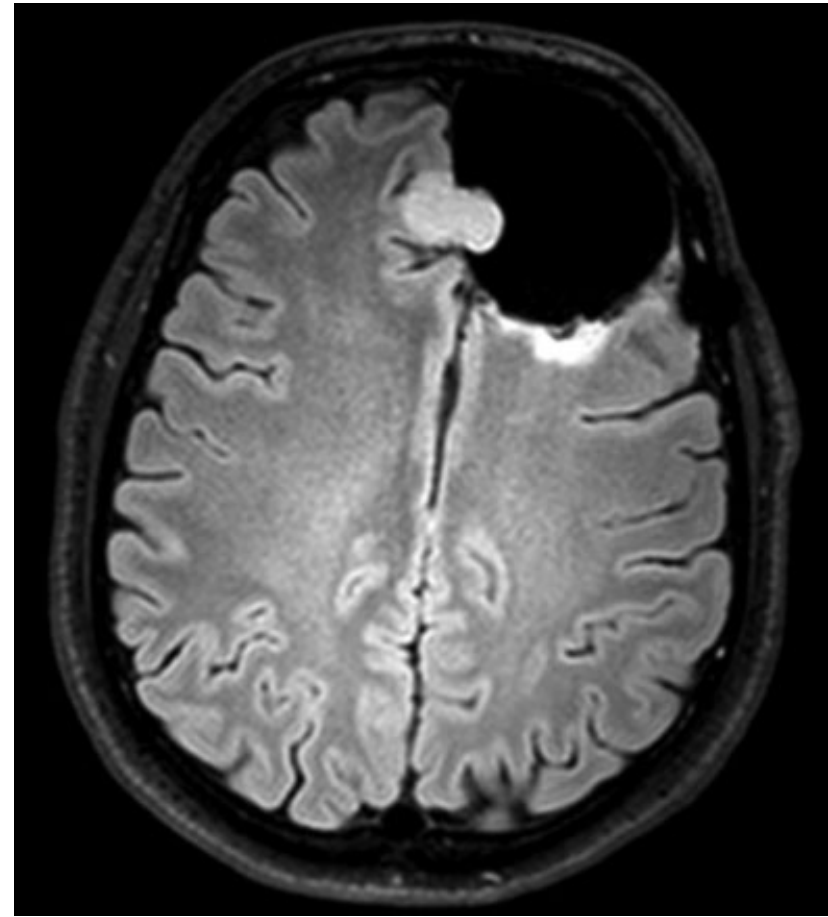

Fig. 7 T2-fluid-attenuated inversion recovery (FLAIR) magnetic resonance imaging showed a tumor recurrence in the posterior and medial walls of the surgical site.

indications for adjuvant treatments, given the clinical, imaging, anatomopathological and degree of tumor removal obtained.

After 12 months, new MRI control showed tumor recurrence, now with a contrast enhancement area, suggesting

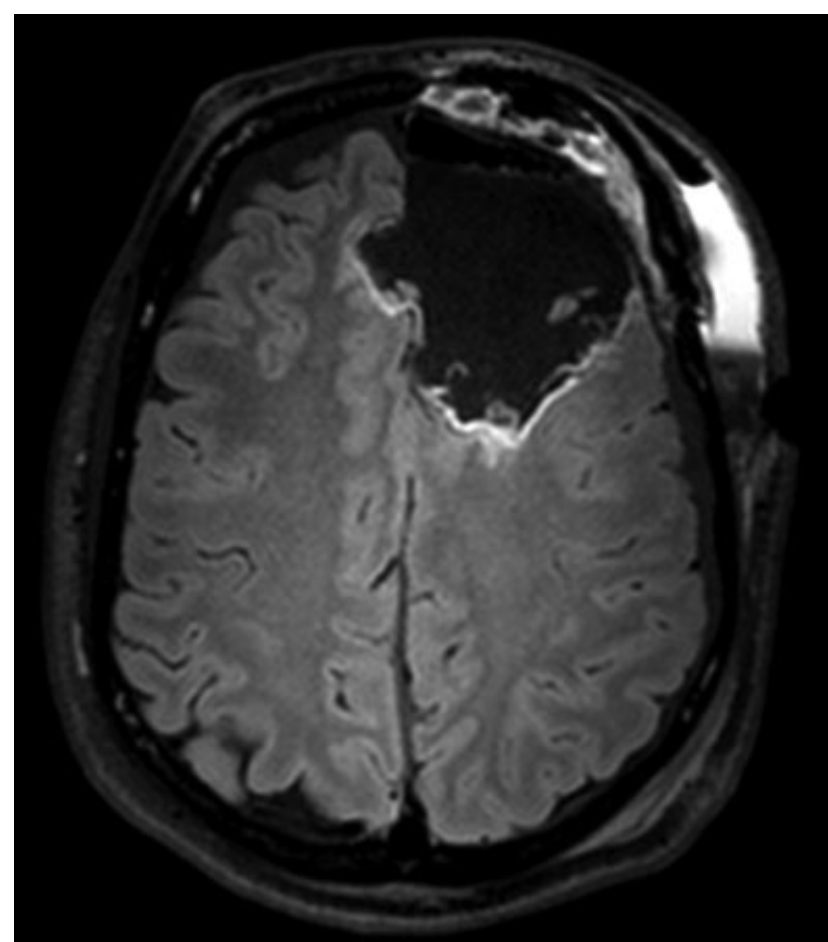

Fig. 8 Second surgery postoperative magnetic resonance imaging showed gross total resection of the tumor. 


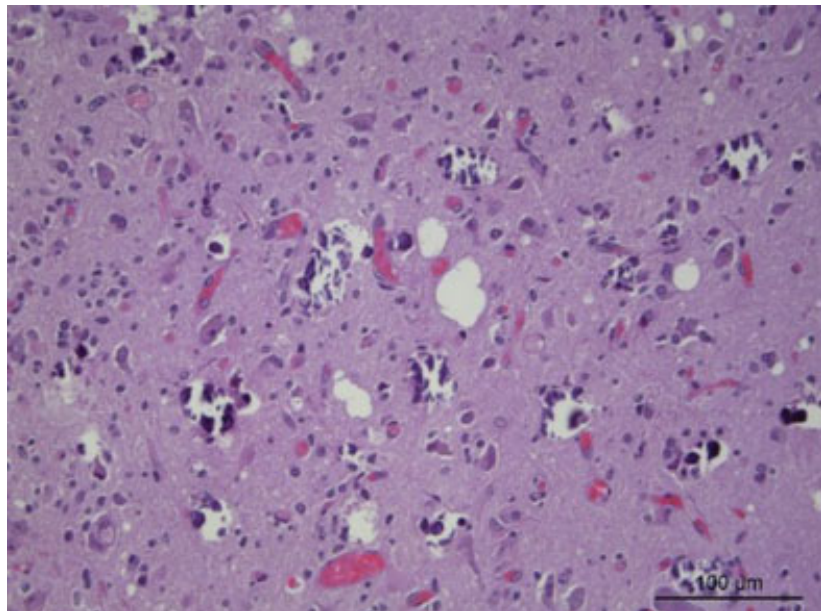

Fig. 9 Hematoxylin \& eosin staining with de same initial type of cells, but with a moderate proliferation index.

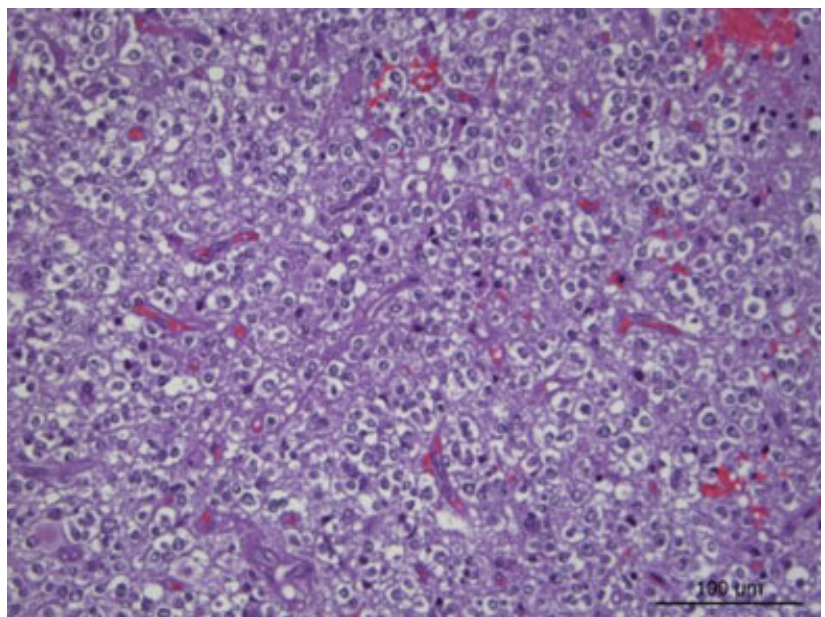

Fig. 10 Hematoxylin \& eosin staining with de same initial type of cells, but with a moderate proliferation index.

possible dedifferentiation (-Figs. 11 and 12). It was operated on with a GTR of the lesion, as evidenced by postoperative brain CT (-Fig. 13). The histological analysis showed a very cellular tumor, with frequent mitoses, areas of extensive necrosis, with rounded nuclei cells, and an evident cytoplasm and a fasciculated aspect. The immunohistochemistry study showed positivity for glial fibrillary acidic protein (GFAP), and the sarcomatous portion was strongly positive for vimentin. It maintained positivity for IDH-1 and ATRX and presented a very high proliferation index. The neuropathological study now showed the occurrence of sarcomatous transformation, maintaining the oligodendroglial component (oligosarcoma WHO grade III ${ }^{4}$ ), (- Figs. 14-20). With this new histological diagnosis, the patient started complementary treatment according to the Stupp protocol. He completed 30 sessions of radiotherapy ( $2 \mathrm{~Gy} /$ session, totalizing $60 \mathrm{~Gy}$ ), with concomitant temozolomide $75 \mathrm{mg} / \mathrm{m}^{2}$ for 7 days/week, for 6 weeks. Following this, the patient underwent 1 cycle of adjuvant temozolomide $150 \mathrm{mg} / \mathrm{m}^{2}$ for 5 days.

During the 2nd cycle of chemotherapy, the patient presented with a new neurological deficit, a motor apraxia of the

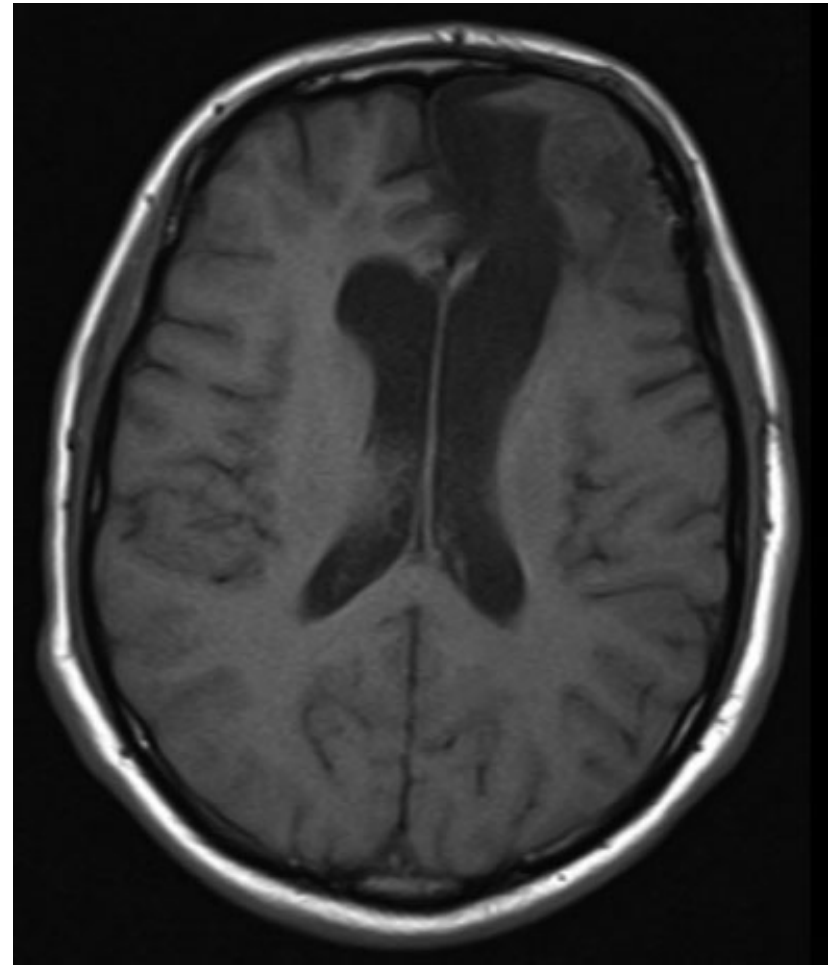

Fig. 11 T1-weighted magnetic resonance imaging showed a left frontal lesion with contrast enhancement (12).

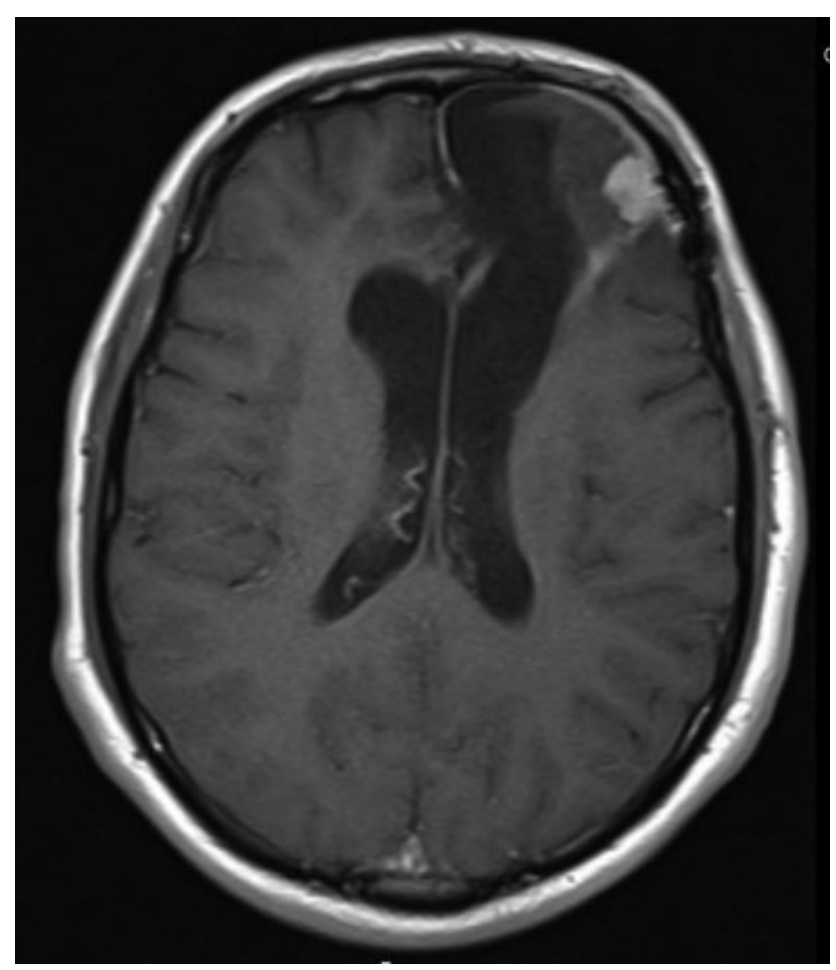

Fig. 12 T1-weighted magnetic resonance imaging showed a left frontal lesion with contrast enhancement (12).

right upper limb. Another MRI was undertaken, which revealed tumor progression, with a recurrent tumor in the surgical site and still with left hemispheric meningeal dissemination revealing an extra-axial mass with contrast 


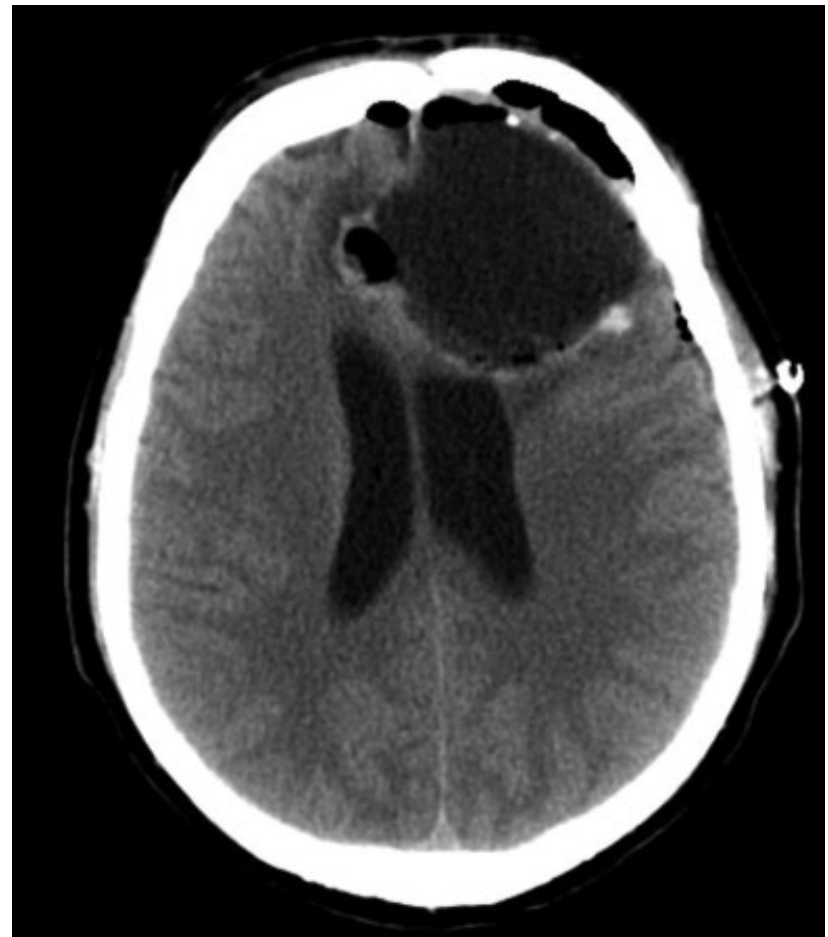

Fig. 13 Third surgery postoperative computed tomography scan without contrast, showed gross total resection of the tumor.

enhancement (-Figs. 21 and 22). A new surgical intervention with GTR of the various relapsed lesions, as documented by postoperative MRI ( $\mathbf{- F i g s . 2 3}$ and $\mathbf{2 4}$ ), was performed. The parietal lesion corresponded to a mass of hard consistency and was extra-axial (-Figs. 25 and 26). The neuropathological study of the various lesions was shown to be the same sarcomatous tumor, and complementary treatment with 2nd line chemotherapy (irinotecan with bevacizumab) was initiated. At the end of 2 months of treatment, the patient exhibited severe cervical radiculopathy without relief with

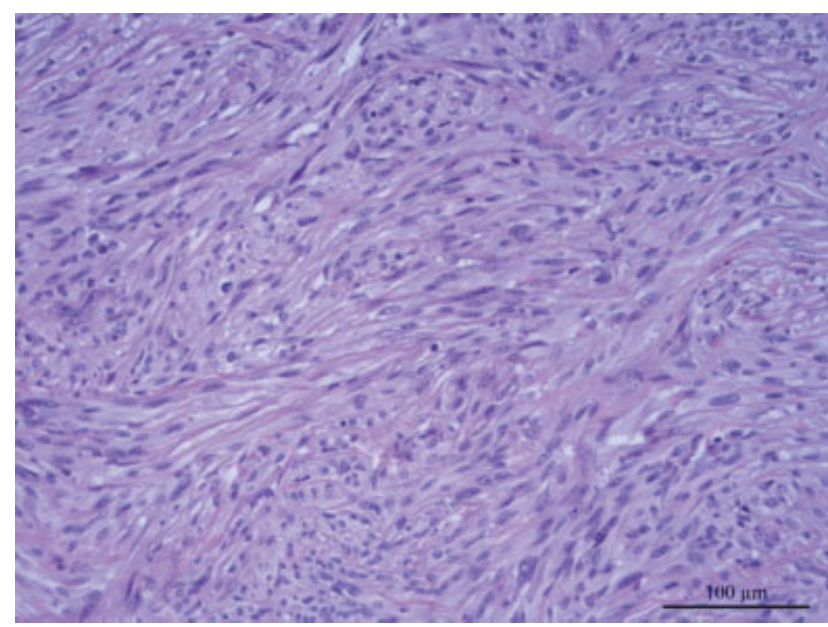

Fig. 14 Hematoxylin \& eosin staining showed hypercellular tumor with round nuclei, with a perinuclear halo, with frequent mitoses and areas of extensive necrosis.

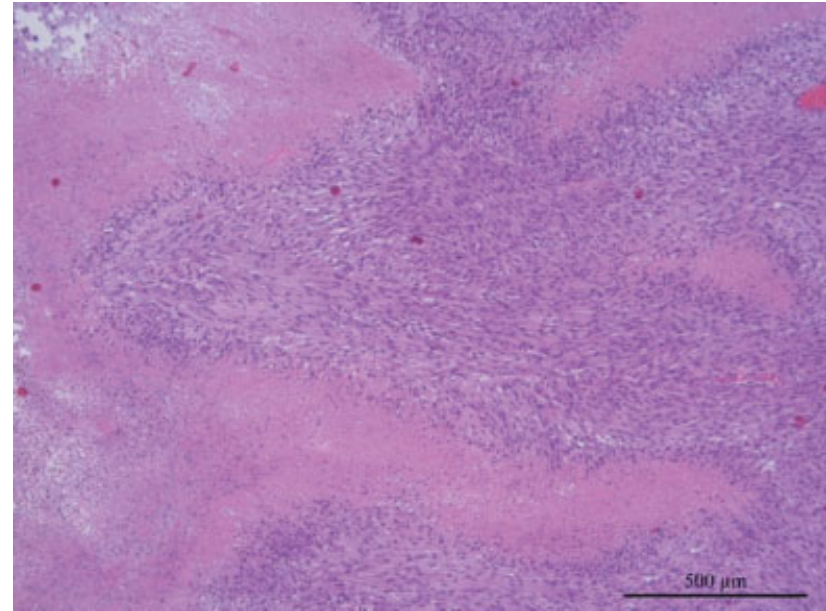

Fig. 15 Hematoxylin \& eosin staining showed hypercellular tumor with round nuclei, with a perinuclear halo, with frequent mitoses and areas of extensive necrosis.

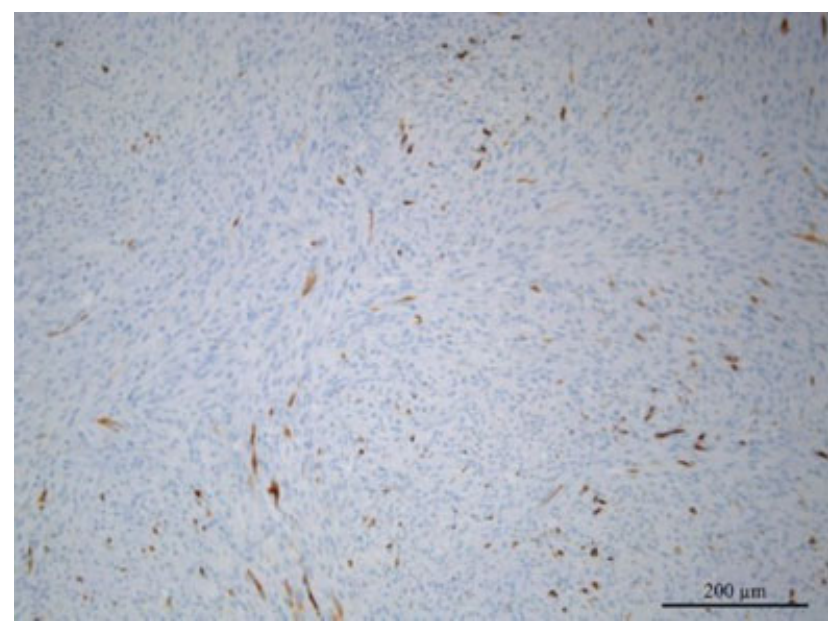

Fig. 16 Immunohistochemistry showed positive staining for glial fibrillary acidic protein (GFAP).

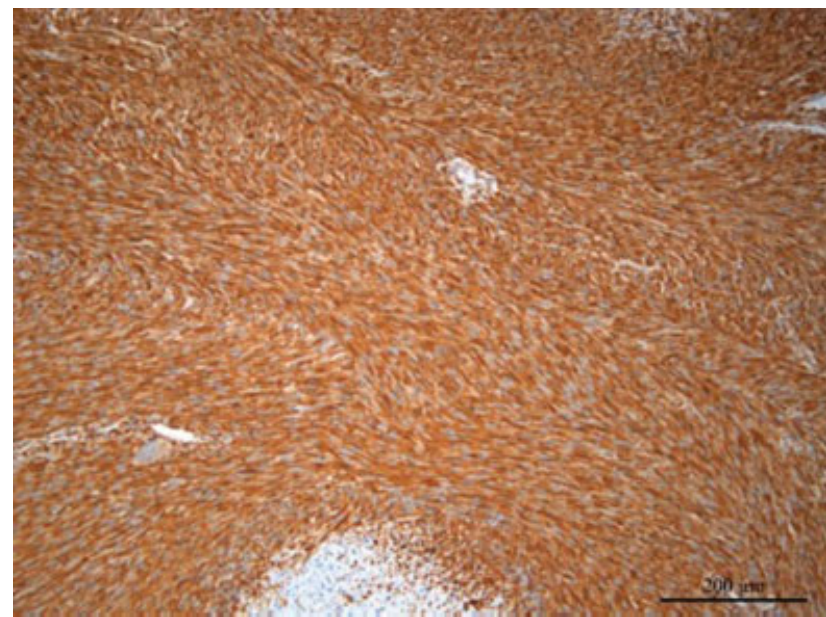

Fig. 17 The tumor showed positive staining for vimentin in the sarcomatous portion. 


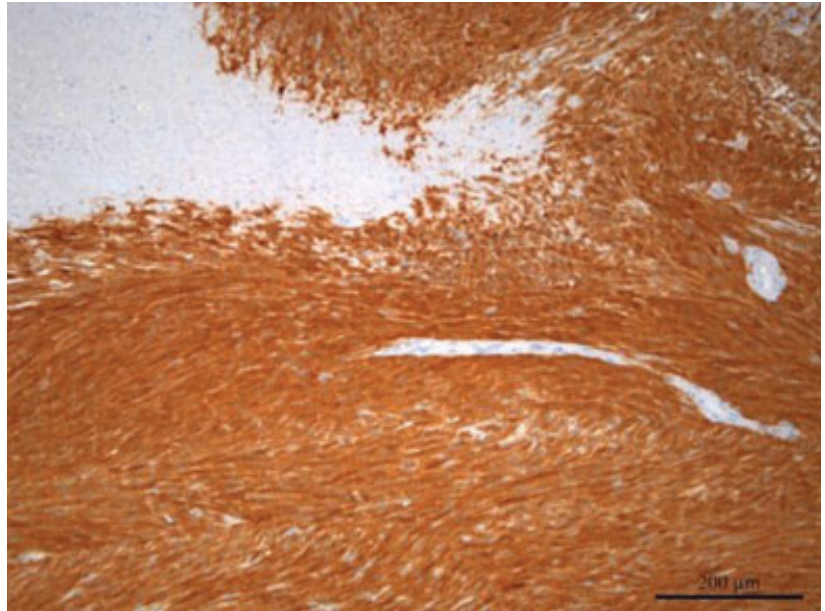

Fig. 18 The tumor was positive for IDH-1.

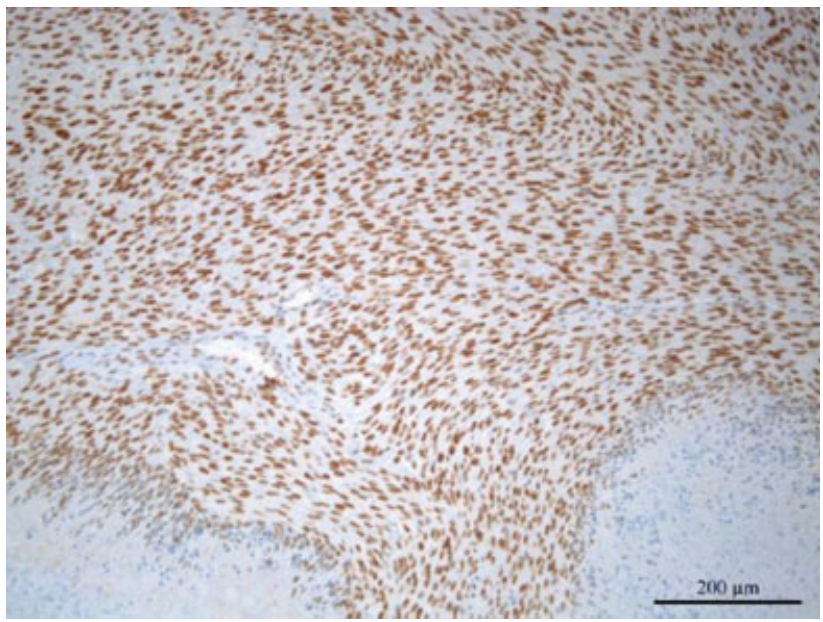

Fig. 19 The tumor was positive for $\alpha$ thalassemia/mental retardation syndrome X-linked (ATRX).

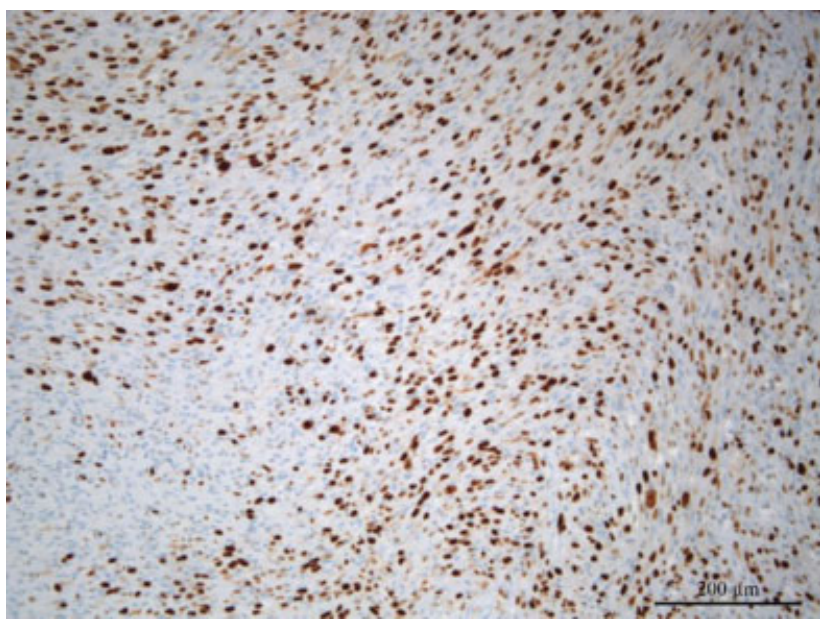

Fig. 20 The proliferation index was very high.

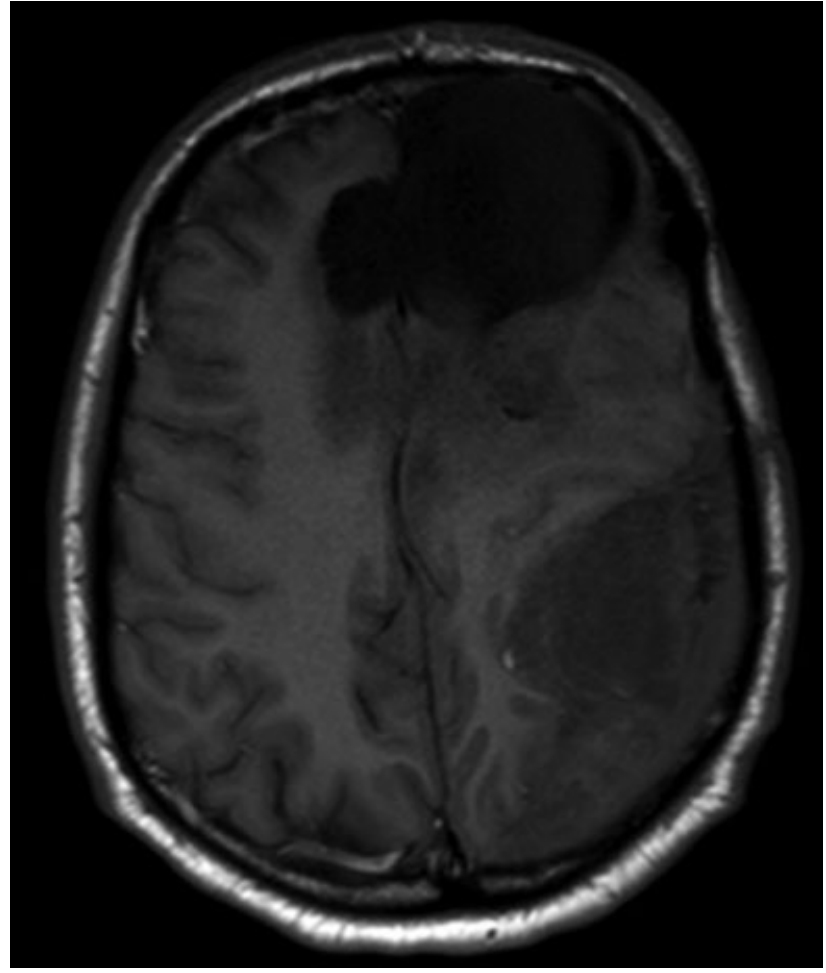

Fig. 21 T1-weighted magnetic resonance imaging showed a recurrent tumor in the surgical site and a left hemispheric extra-axial mass with contrast enhancement (22).

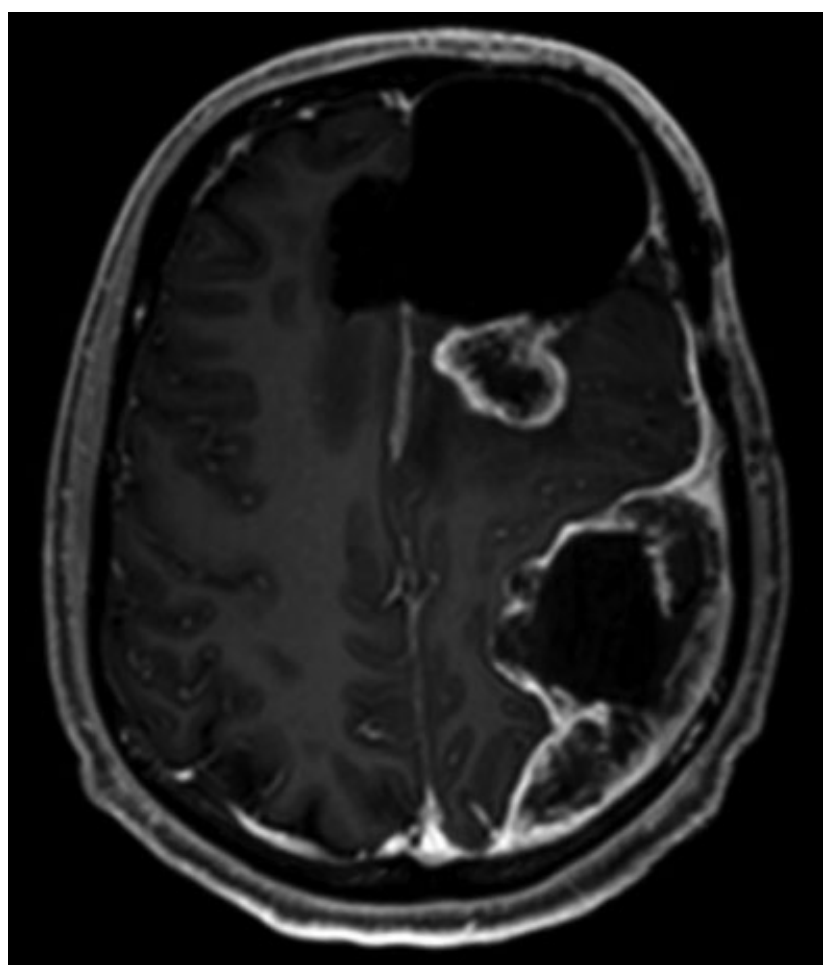

Fig. 22 T1-weighted magnetic resonance imaging showed a recurrent tumor in the surgical site and a left hemispheric extra-axial mass with contrast enhancement (22). 


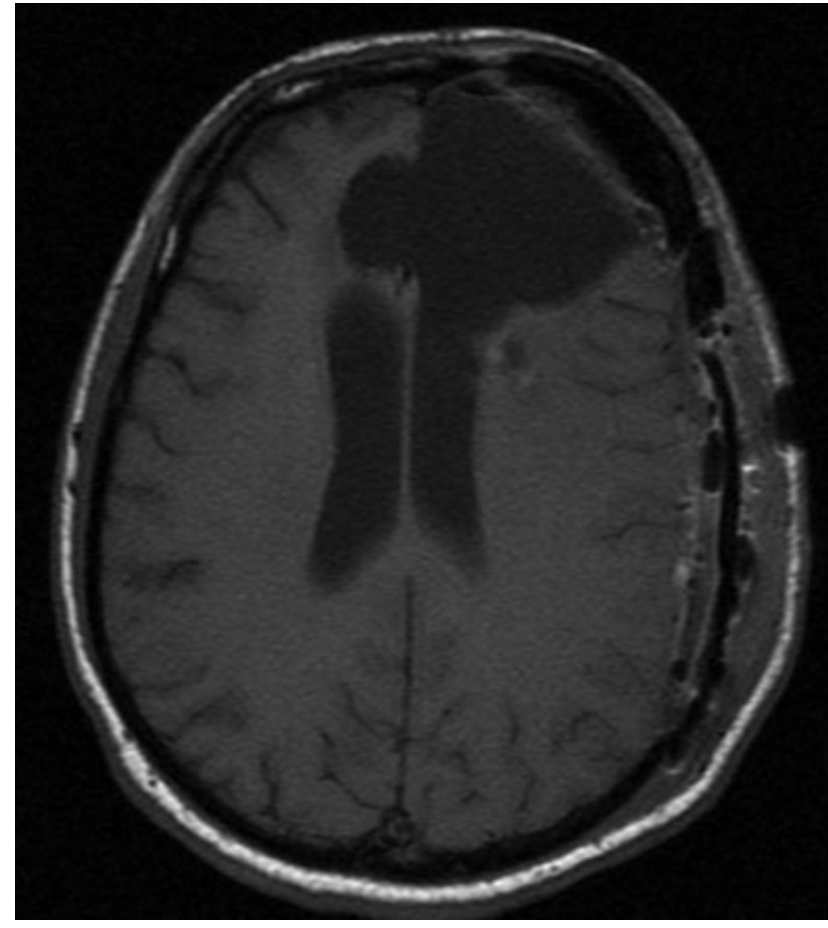

Fig. 23 Fourth postoperative magnetic resonance imaging showed gross total resection of the tumor.

analgesics, and was submitted to lumbar puncture, which showed the presence of malignant cells in the cerebrospinal fluid. He underwent a neuro-axis MRI, which showed stability of the disease at the brain but extensive spreading to the medulla with intradural and extramedullary lesions at the

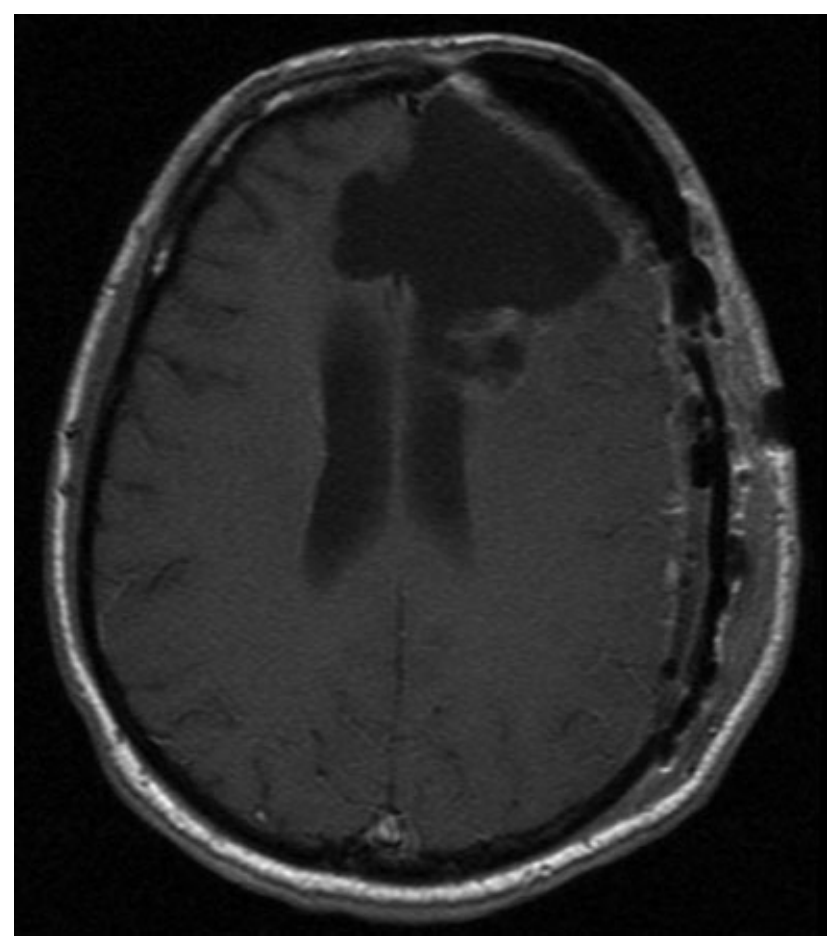

Fig. 24 Fourth postoperative magnetic resonance imaging showed gross total resection of the tumor.

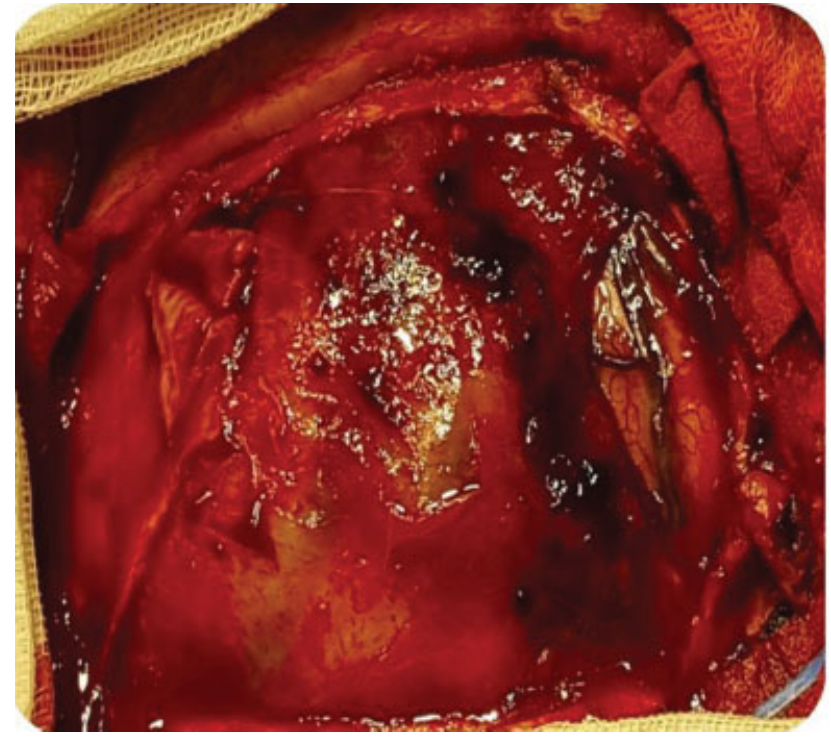

Fig. 25 Intraoperative images showing a lesion of hard consistency, with plane of separation of adjacent brain parenchyma.

cervical and medullary cone levels ( - Figs. 27, 28). He started treatment with 3rd line chemotherapy (lomustina $90 \mathrm{mg} / \mathrm{m}^{2}$ for 6 days/week, for $/ 6$ weeks, and bevacizumab $10 \mathrm{mg} / \mathrm{kg}$ for 2 days/week for 2 weeks. The patient also performed 5 sessions of radiotherapy directed to the cervical lesion (5 fractional radiotherapy sessions totaling $20 \mathrm{~Gy}$ ). Currently, the patient has a 4-year overall survival, with Karnofsky performance status of $60 \%$ and eastern cooperative oncology group (ECOG) performance status 2 .

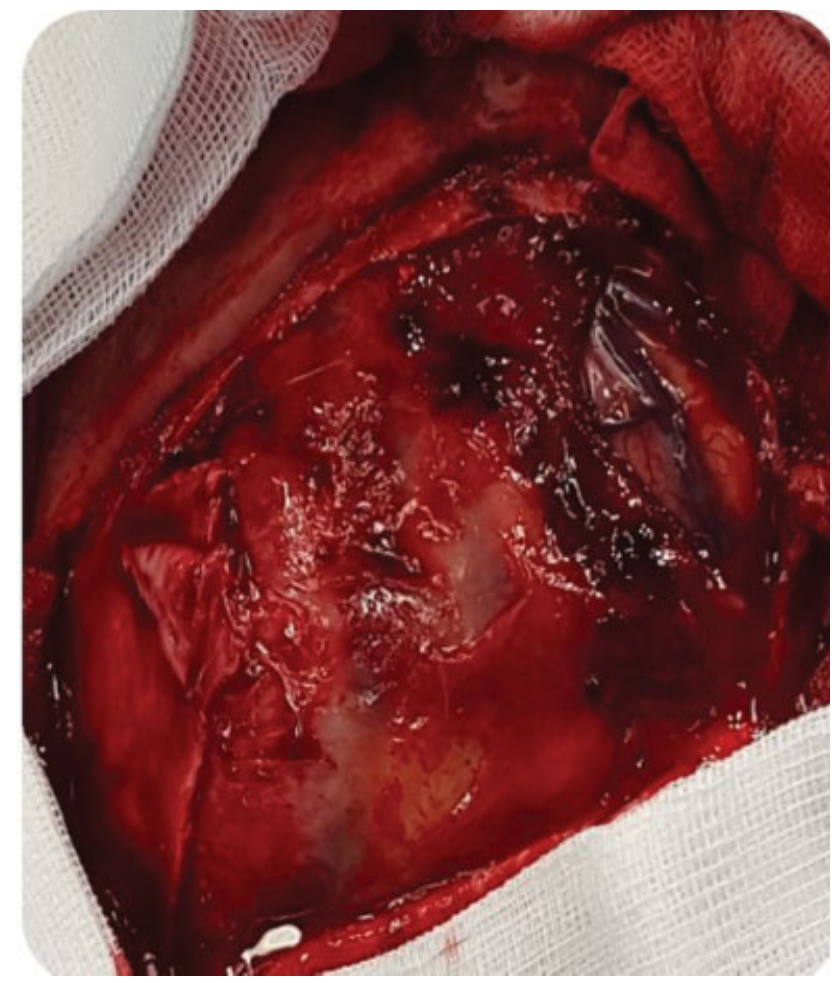

Fig. 26 Intraoperative images showing a lesion of hard consistency, with plane of separation of adjacent brain parenchyma. 


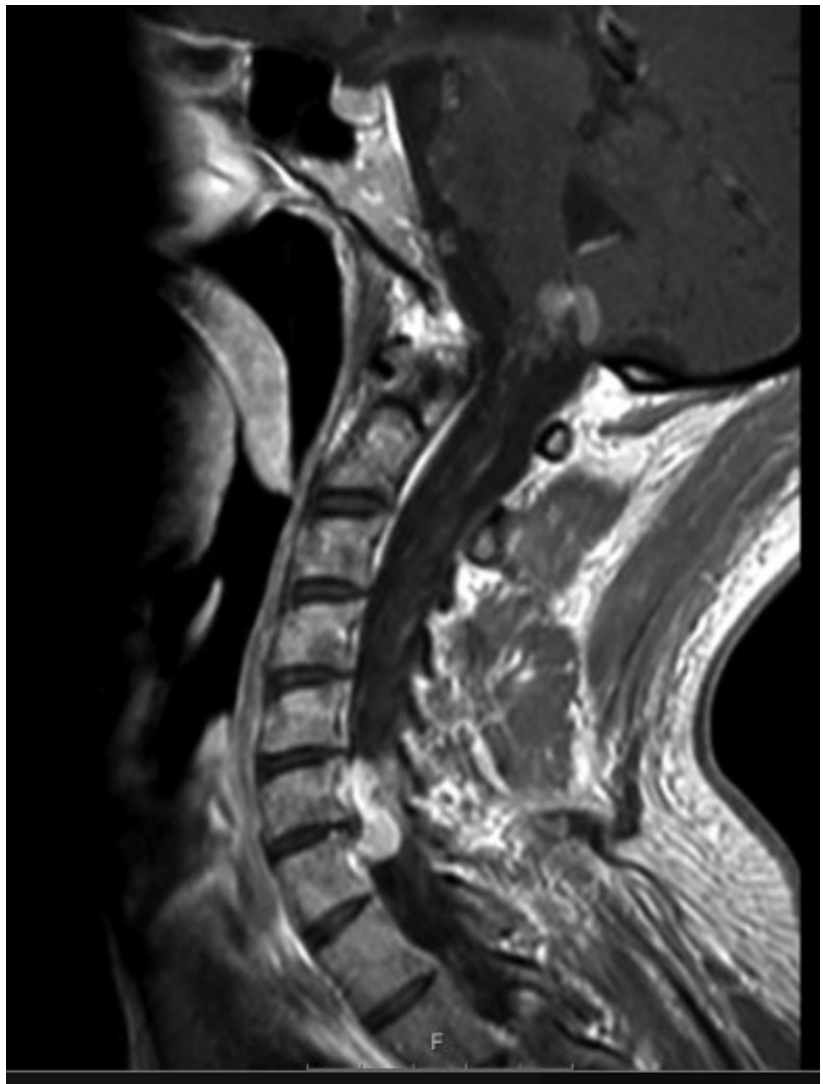

Fig. 27 Cervical T1-weighted magnetic resonance imaging with contrast showed a lesion at C6-C7 level with contrast enhancement.

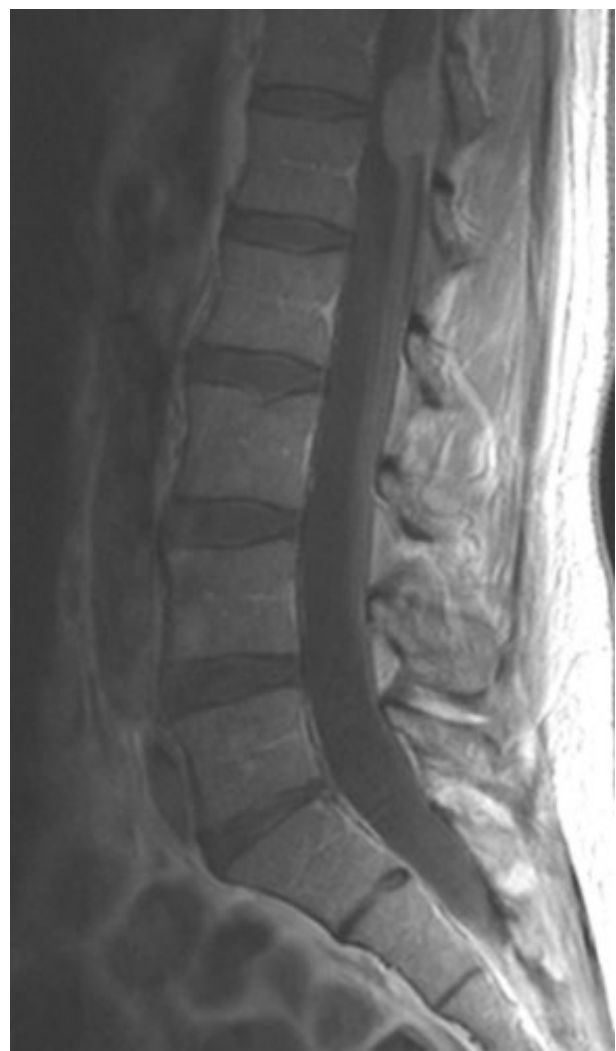

Fig. 28 Lumbar T1-weighted magnetic resonance imaging with contrast showed a lesion at T12-L1 level with contrast enhancement.

\section{Discussion}

Isocitrate dehydrogenase-mutated and $1 \mathrm{p} / 19 \mathrm{q}$-positive codeletion oligodendrogliomas are considered slow-growing tumors with a better prognosis than the other gliomas. ${ }^{5}$ The appearance of sarcomatous tumors at sites of oligodendrogliomas resection in patients not undergoing further chemotherapy and/or radiotherapy treatments is very rare. ${ }^{6}$ Although in most cases the glial component of the sarcomatous tumors is astrocytic, the literature describes several cases of gliosarcomas in which the glial component is oligodendrocytic. ${ }^{2,3,6-10}$

Here, we describe the case of an oligodendroglioma WHO grade II with 1p/19q co-deletion, IDH-1 and ATRX mutation, with initial GTR, not subjected to complementary treatments, and which was dedifferentiated to the sarcomatous form. The tumor always maintained the same genetic characteristics. Despite the presence of predictive factors of better prognosis, the tumor displayed poor response to radiotherapy and chemotherapy, and even presented spinal metastasis. Although several cases have been described in the literature of oligodendrogliomas with transformation to the sarcomatous form (oligosarcoma), research performed in PubMed and Google Scholar reveals only one case with spinal metastasis. ${ }^{8}$

Most cases with extracranial dissemination are associated with extensive progression of the brain tumor; ${ }^{11}$ yet, in this case, the spread occurred with stability of the brain lesions.

This case describes a patient with a low-grade glioma, with most of the predictive factors of better prognosis (age $<40$ years, total initial resection and favorable genetics), in whom progression occurred rapidly and with refractoriness to complementary treatments.

\section{Conclusion}

Despite all the good prognostic factors present in this clinical case and the absence of previous adjuvant therapies, the tumor was dedifferentiated to a malignant form, quickly and without any warning signs.

This leads us to conclude that there is a need for further studies that may indicate new prognostic factors, such as imaging, anatomopathological and genetic characteristics that help us understand which tumors will dedifferentiate more quickly and which may respond better to complementary treatments.

\section{Conflicts of Interest}

The authors have no conflicts of interest to declare.

\section{References}

1 Paleologos NA, Cairncross JG. Treatment of oligodendroglioma: an update. Neuro-oncol 1999;1(01):61-68

2 Vajtai I, Vassella E, Hewer E, Kappeler A, Reinert MM. Sarcomatous evolution of oligodendroglioma ("oligosarcoma"): confirmatory report of an uncommon pattern of malignant progression in oligodendroglial tumors. Pathol Res Pract 2012; 208(12):750-755 
3 Rodriguez FJ, Scheithauer BW, Jenkins R, et al. Gliosarcoma arising in oligodendroglial tumors ("oligosarcoma"): a clinicopathologic study. Am J Surg Pathol 2007;31(03):351-362

4 Louis DN, Ohgaki H, Wiestler OD, Cavenee WK. 2016World Health Organization Histological Classification of Tumours of the Central Nervous System. International Agency for Research on Cancer, France

5 Louis DN, Ohgaki H, Wiestler OD, Cavenee WK. 2007World Health Organization histological classification of tumours of the central nervous system. International Agency for Research on Cancer, Lyon

6 Yasuda T, Nitta M, Komori T, et al. Gliosarcoma arising from oligodendroglioma, IDH mutant and $1 \mathrm{p} / 19 \mathrm{q}$ codeleted. Neuropathology 2017;•..;. Doi: 10.1111/ neup.12406

7 Shoji T, Saito R, Kanamori M, Sonoda Y, Watanabe M, Tominaga T. Sarcoma-like tumor originating from oligodendroglioma. Brain
Tumor Pathol 2016;33(04):255-260. Doi: 10.1007/s10014-0160268-2

8 Pasquier B, Couderc P, Pasquier D, Panh MH, N'Golet A. Sarcoma arising in oligodendroglioma of the brain: a case with intramedullary and subarachnoid spinal metastases. Cancer 1978;42(06): 2753-2758

9 Hiniker A, Hagenkord JM, Powers MP, Aghi MK, Prados MD, Perry A. Gliosarcoma arising from an oligodendroglioma (oligosarcoma). Clin Neuropathol 2013;32(03):165-170

10 Tanaka S, Hitotsumatsu T, Sugita Y, et al. Gliosarcoma arising from oligodendroglioma (Oligosarcoma): A case report with genetic analyses. Pathol Int 2018;68(10):567-573

11 Choi TM, Cheon YJ, Jung TY, Lee KH. A Stable Secondary Gliosarcoma with Extensive Systemic Metastases: A Case Report. Brain Tumor Res Treat 2016;4(02):133-137 